\title{
NR Sidelink Enhancement in 3GPP Release 17
}

\author{
Karthikeyan Ganesan*, Prateek Basu Mallick and Joachim Löhr \\ Lenovo, Oberursel, Germany \\ E-mail: kganesan@lenovo.com; pmallick@lenovo.com; jlohr@lenovo.com \\ ${ }^{*}$ Corresponding Author
}

Received 27 November 2020; Accepted 18 February 2021;

Publication 26 May 2021

\begin{abstract}
3GPP is currently studying enhancements to Sidelink (SL) operations for $5 \mathrm{G}$ New Radio (NR) in a Release 17 Work Item which is planned to be finished by end of 2021. The NR Sidelink (SL) Work Item in Release 17 includes several key features targeting reliability enhancements, power saving and coverage enhancements by expanding the scope of NR sidelink to target V2X, commercial D2D use-case and Public safety. This paper provides an insight on the current 3GPP Release 17 NR SL design describing necessary enhancements in the physical, protocol layer to support inter-UE coordination message for reliability enhancement for autonomous resource selection procedure by providing feedback on the half-duplex, persistent collision and hidden nodes. In addition, the power saving feature is addressed by introducing a SL DRX mechanism for the PC5 interface which defines active reception and transmission periods between a TX and the peer $\mathrm{Rx}$ $\mathrm{UE}(\mathrm{s})$ and also between Tx UE and gNB. Furthermore, this paper outlines details on the Sidelink Relay feature for coverage enhancement by describing possible solutions for UE to Network relay and UE to UE relay.
\end{abstract}

Keywords: NR Sidelink, Inter-UE coordination, SL DRX, UE to UE Relay.

Journal of ICT Standardization, Vol. 9_2, 79-90. River Publishers

doi: 10.13052/jicts2245-800X.922

This is an Open Access publication. () 2021 the Author(s). All rights reserved. 


\section{Introduction}

3GPP standardization is evolving beyond $5 \mathrm{G}$ the New Radio air interface (NR-Uu) framework defined in 3GPP Release 15 (also known as 5G phase 1) to study on the support of $5 \mathrm{G}$ for vertical industries like automotive, manufacturing industry etc., 3GPP Release 16 (5G phase 2) finalized at the end of 2019 introduced physical layer, protocol and core network functionalities supporting NR sidelink for different cast types i.e., Broadcast, Groupcast, Unicast [6]. The advanced V2X services primarily focuses on supporting platooning, advanced driving, extended sensor and remote driving use-cases and these advanced V2X services need demanding reliability, lower latency, enhanced coverage while power saving feature is essential to support pedestrian/vulnerable road users [7]. Many of the NR sidelink physical layer and protocol features developed based on the V2X requirements can be reused to support commercial D2D use cases, public safety etc., 3GPP Release 17 standardization is currently investigating the NR sidelink enhancement to support higher reliability for $\mathrm{V} 2 \mathrm{~V}$ service, power saving features for Pedestrian/Vulnerable Road Users, commercial D2D use-cases etc.

NR Release 16 sidelink is designed with multicarrier waveform i.e., Cyclic-Prefix Orthogonal Frequency-Division Multiplexing (CP-OFDM) compared with LTE sidelink design of single carrier waveform. NR Release 16 sidelink design for frequency range 1 (FR1) which is up-to $6 \mathrm{GHz}$ supports Subcarrier Spacing (SCS) like $15 \mathrm{KHz}, 30 \mathrm{KHz}$ and $60 \mathrm{KHz}$. For FR2 ranging from $6 \mathrm{GHz}$ to $52.6 \mathrm{GHz}$ Subcarrier Spacing of $60 \mathrm{KHz}, 120 \mathrm{KHz}$ are supported. The Extended Cyclic Prefix (ECP) support is added only for $60 \mathrm{KHz}$ SCS providing robustness against high delay spread. The support of higher Subcarrier Spacing in the system ensures robustness against Doppler [1, 3].

The Physical Sidelink Control Channel (PSCCH) carrying Sidelink Control Information (SCI) is time multiplexed with the associated Physical Sidelink Shared Channel (PSSCH) whereas in LTE sidelink it is multiplexed in frequency domain. Another major difference in the physical layer design is the introduction of two stage SCI where the content of the SCI payload is separated into two parts. The first SCI containing sensing information is broadcasted to the surrounding UEs containing information related to QoS priority of data, occupied resource blocks, resource reservation interval etc., whereas the second SCI transmission carries information related data decoding of Physical SL Shared Channel (PSSCH) [2]. The higher reliability of NR

Release 16 sidelink for unicast and groupcast transmission is achieved with the introduction of the new Physical Sidelink Feedback Channel (PSFCH) 


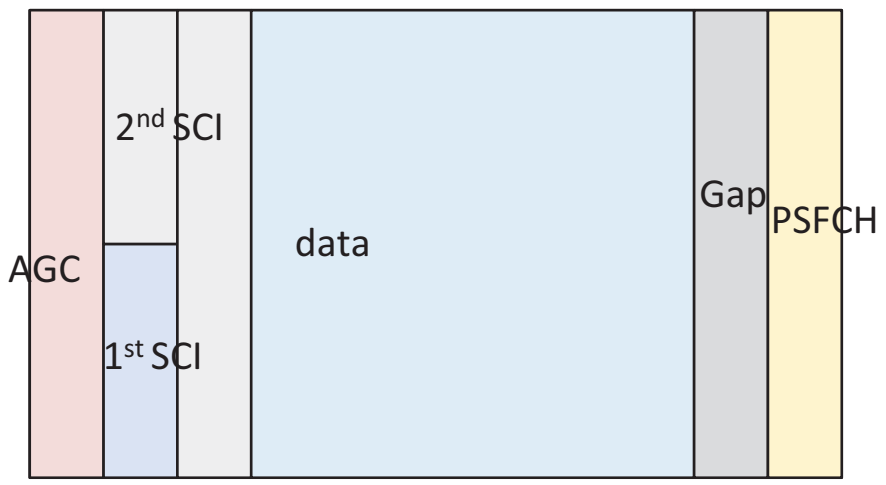

Figure 1 NR Sidelink frame structure.

to carry HARQ feedback in the last OFDM symbol of a slot as shown in Figure 1. In order to perform efficient link adaptation for unicast transmissions, feedback of the channel quality information (CQI) is supported from the Receiver (RX) UE.

NR Release 16 sidelink supports two HARQ feedback options for groupcast considering reliability and feedback resource efficiency. In the first option (Option-1), when receiver UE fails to decode a Transport Block (TB) it transmits a HARQ-Negative Acknowledgement (NACK) on a common feedback resource. SCI from Tx UE includes both the Minimum communication Range (MCR) parameter and its present location in the form of a Zone id. This information is further used by RX UEs to calculate the Tx-Rx distance and determines about the transmission of HARQ feedback when the Tx-Rx distance is less than the MCR. In the second option (Option-2), receiver UE transmits on a dedicated feedback resource allocated for each Rx UE(s) in a group, HARQ-Acknowledgement (ACK) or HARQ-NACK according to the decoding status of its TB.

NR Release 16 sidelink supports two modes of resource selection methods where in the first method named as NR Mode 1, gNB centrally allocates resources for sidelink communication thereby requiring a sidelink Tx UE to interface with gNB for sidelink resource request/reception before transmitting on the PC5 interface and in the second method named as NR Mode 2, sidelink UE autonomously selects sidelink resources from a pre-configured resource set for transmission over sidelink and transmitter UE selects a suitable vacant resource with the help of sensing thereby monitoring Sidelink Control Information (SCI) from all nearby transmitting UEs. 


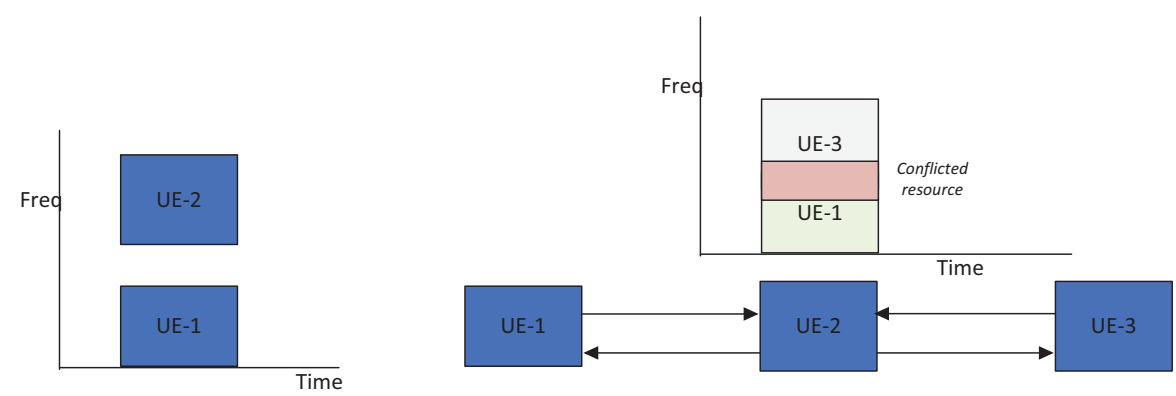

Figure 2 Shows Half duplex and Hidden node issue.

In general, compared to the gNB based resource selection method for sidelink, the reliability of the NR sidelink devices are affected for distributed and autonomous resource selection methodology due to resource collision, half duplex and hidden nodes. Reliability of the sidelink devices are affected due to half duplex constraints where a transmitting sidelink device cannot receive data at the same time slot from neighbouring sidelink devices, secondly reliability is affected due to problem with persistent collision when more than one nearby sidelink device selects the same time/frequency resource for transmission. Another problem affecting the reliability of the sidelink communication is caused by hidden nodes where the transmitter device is not aware about the interference at the receiver. NR Release 16 sidelink does not detect consecutive packet failure which is necessary for the enhanced reliability required by some of the sidelink use cases $[4,5]$.

Moreover, NR Release 16 sidelink does not include power saving feature which is essential for Pedestrian UEs/Vulnerable Road Users, commercial D2D use cases and Public safety UEs. Limiting the SCI monitoring slots and introducing DRX sleep period can have a profound influence on extending the battery life of these devices.

Extended sidelink coverage enhancements are needed for the platooning use case, where the communication from the platoon leader is communicated to all members belonging to a platoon.

This paper presents an overview of potential technical solutions studied and considered under Release 17 NR SL to meet the requirements of enhanced reliability, power saving and coverage enhancement. At the time of writing this paper, Release 17 standardization is just started and hence solutions described in this paper are from the viewpoint of author. The effort of facilitating an ultra-reliable NR sidelink by preventing consecutive packet loss and the introduction of power saving, Relaying is critical for realizing all V2X communication services, which are paramount for road safety. 
The structure of the paper is as follows, in Section 2, we introduce the NR SL physical layer enhancements for Rel-17. Section 3 describes SL protocol enhancement as part of power saving feature and then Section 4 describes the Relaying functionality. Finally, we conclude in Section 5 providing a brief discussion on the outlook.

\section{NR SL Physical Layer Enhancements for Rel-17}

The decentralized nature of the sidelink communication results in collisions due to the random nature of resource selection, in which more than one UEs could select overlapping resources. In NR Release 16 sidelink, Tx UE can reserve up to a maximum of 3 resources within a window of 32 slots and the reserved resource is signalled within the first stage SCI. Collisions happens in a reserved resource once a sidelink UE broadcast the reserved resource using its first stage SCI while nearby UEs fail to receive or decode the SCI containing the UE's reservation information, resulting in more than one UE transmitting on overlapping resources. In typical urban scenario such as intersection or crossroad where there are blockages due to buildings, although UEs are closer to each other but due to Non-Line of sight (NLOS) the measured RSRP likely remains low. In such cases, the resource is not likely excluded in the candidate resource selection procedure since the measured RSRP is likely below the configured RSRP threshold for the resource exclusion. These failures affect the reliability in sidelink communication and can be due to the hidden node problem, half duplex constraints, or processing limitations. Such failure can be fundamentally avoided using additional assistance information such as inter-UE coordination message from neighbouring UEs.

\subsection{Inter-UE Coordination}

Exchanging of assistance information in NR Release 17 sidelink can help with both pre-collision and post-collision detection as shown in Figure 3. Pre-collision is usually detected by a UE with decoding the reserved resource information in the first stage SCI. The reliability issue arising due to a potential half duplex constraint or resource collision in a sidelink communication can be successfully mitigated with the help of UEs periodically monitoring the SCI from nearby UEs and providing necessary feedback. UE can additionally monitor the destination group identifier transmitted in the SCI along with the resource information for pre-collision and post-collision detection, by providing feedback to group member UEs about a potential half duplex problem or resource collision problem. 


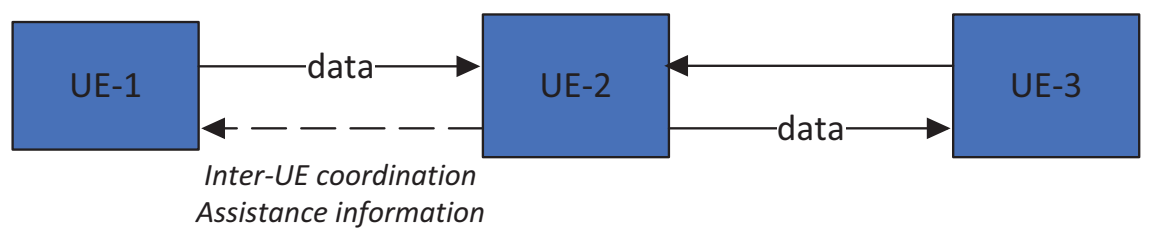

Figure 3 Inter UE coordination message.

Assistance information such as inter-UE resource coordination message by transmitting a preferred or not preferred/conflicted set of resource can help in the candidate resource selection procedure of transmitter UE either by considering the preferred resources otherwise avoiding those not preferred/conflicted resource. Transmitter UE can also perform joint resource selection by considering its own preferred candidate resource together with the preferred resource set received from other UE. A UE can trigger resource (re)selection mechanism to reselect the reserved resource transmitted in the first stage SCI once it receives the inter-UE coordination message containing not preferred/conflicted set of resource from other UE.

There can be different methods for exchanging inter-UE coordination message such as aperiodic explicit trigger for the transmission of inter-UE coordination message, periodic transmission of coordination message and event-trigger approach. In the first method, Transmitter UE can request other UE for the transmission of inter-UE coordination message by transmitting a trigger in the SCI. Such aperiodic triggering and reporting of inter-UE coordination message is helpful in identifying and recovering from failures happened in the past. In second method, periodic transmission of inter-UE coordination is helpful in reducing the probability of collision beforehand. In the third method of event triggered approach is based on the consecutive NACKs at the receiver UE helping in reducing successive packet reception failure.

\section{Power Saving Features}

\subsection{SL DRX Mechanism}

Discontinuous Reception (DRX) mechanism was introduced for the NR Uu interface to prolong UE battery lifetime, by allowing UE to enter sleep mode at regular period by turning off its RF modem, baseband receiver etc as shown in Figure 4. However, in sidelink communication, UE can be implemented with a separate sleep cycle time for reception and transmission. Configuration of separate/individual separate sleep cycle time for reception and separate 
sleep cycle time for transmission might practically turn out to be difficult and inefficient to implement, since the reception and transmissions of data are intrinsically linked in most cases e.g. when a receiver (of a data packet) must also transmit a HARQ feedback in response to the reception of a data packet. Therefore, in a sidelink communication, with more than one UEs involved, 'not receiving' for one UE means also 'not transmitting' for the other. Hence, it is easier and more power efficient to introduce a mechanism allowing sidelink UEs to sleep for both reception as well as for transmission using one single sleep cycle with a resulting "DRX" cycle allowing turn off both its receiver circuitry and transmitter circuitry.

The decentralized nature of the sidelink communication requires new solution to address DRX cycle configuration between more than one sidelink UEs to start communication. A "common DRX cycle configuration" allows each of these sidelink UEs to receive and/or transmit from nearby UEs. Hence the configuration of this common DRX cycle can be depended on the initiated service or application. In this case, each service/application has an associated SL DRX configuration which means sidelink DRX configuration is linked to the PC5 QoS Identifier (PQI) of a service/application or to a PQI-range (to keep the limited number of configurations).

Sidelink DRX configuration consists of a time slot offset and a periodically occurring time-period to monitor reception and transmission of data. Given that the corresponding SL DRX configuration is known (e.g. preconfigured) for a specific service, both the new UE and a potential-peer would know when to wake up to receive or to transmit data on the PC5 interface for that particular service and at least for how long (on duration) each time period. However, sidelink devices can further co-ordinate to extend the active time, or even modify/ agree on another SL DRX configuration.

Further, it is beneficial if the SL and Uu DRX configurations, i.e. Active Time on Uu interface and the Active Time on the PC5 interface, are aligned for a UE having RRC Connection established on $\mathrm{Uu}$ with the gNB. The sidelink UE signals its SL DRX configuration to the gNB allowing the latter to provide an aligned Uu DRX accordingly. There is one common Active Time on the Uu interface which encompasses the PDCCH monitoring for Uu as well as SL related DCI.

A Go-to-sleep mechanism can also be used for the PC5 interface, where a potential transmitter informs its peer receivers that there is no more data available for transmission by allowing UE to enter the sleep period earlier.

Another method is the usage of relaying to save power by using a nearby UE relay to make transmission to another sidelink UE or to a gNB by using low transmit power. DRX cycles needs to aligned between the Tx UE, relay 


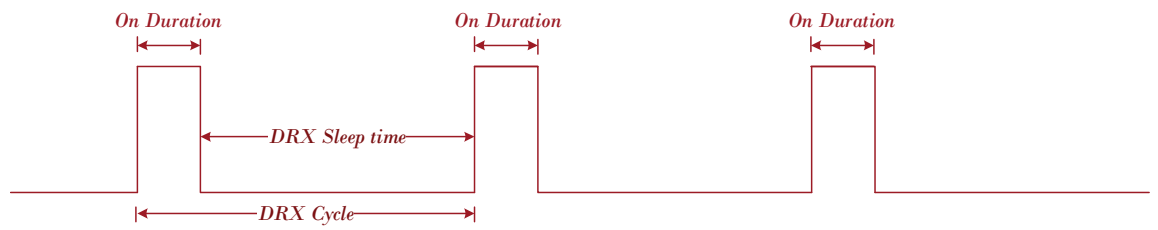

Figure 4 Single DRX Cycle time: UE receives as well as can transmit in the On Duration.

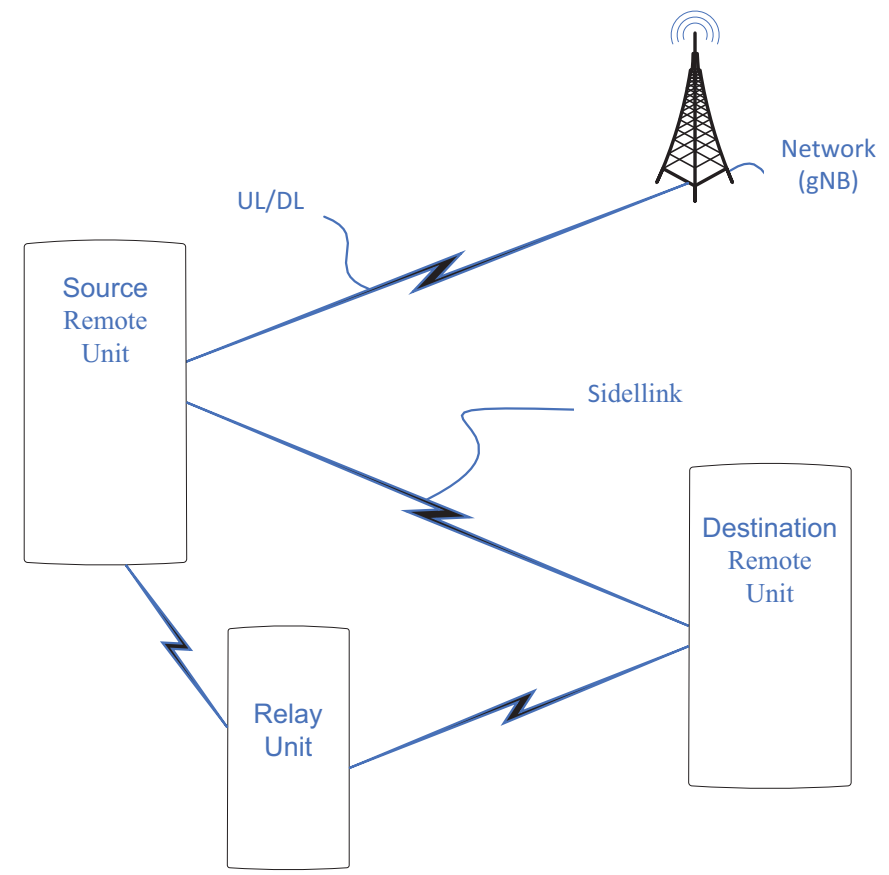

Figure 5 UE-to-network Relay UE and UE-to-UE Relay UE.

$\mathrm{UE}$ and the remote UE to transmit and receive data within the active period, without DRX cycle alignment the relay UE might not be able to receive data from the Tx UE and transmit data to the remote UE.

\section{SL Relay Enhancement - NW Relay and UE-UE Relay}

UE relay as shown in Figure 5 is of two types: UE-to-network Relay UE (U2N Relay) and UE-to-UE Relay UE (U2U Relay). Relay enhances coverage, reliability by using multi-hop or mesh architecture. Tx UE needs 


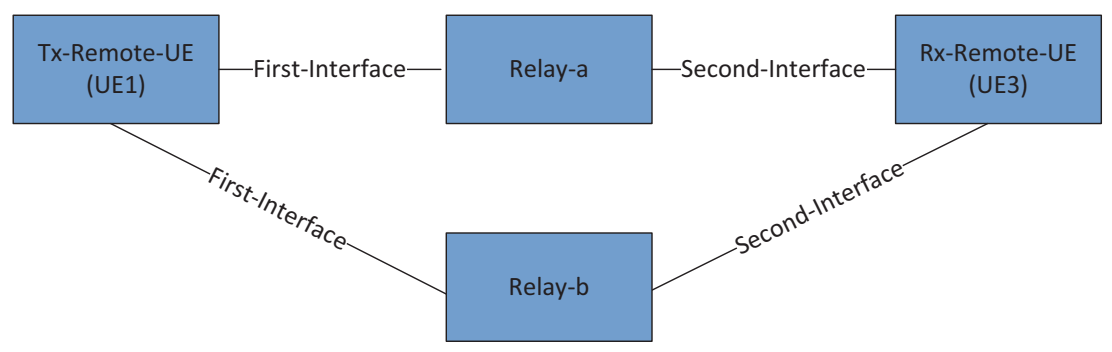

Figure 6 Path selection in a Relay architecture.

to discover and select a Relay before starting its transmissions to a Remote UE. Figure 6 shows an example architecture allowing more paths between the Tx UE and the remote UE 3 for enhancing reliability and coverage. Tx UE can use both relays to transmit data towards the destination remote UE 3 to enhance reliability and then different relay path can be selected in the forward and the reverse link based on the channel condition at the Tx UE and the remote UE.

\section{Conclusion}

This paper outlines the solutions such as inter-UE coordination, DRX and Relaying being discussed in the current NR Rel-17 sidelink for enhancing reliability of NR Mode 2 resource selection, power saving for pedestrian/vulnerable road user/commercial sidelink devices and then for coverage extension respectively. Assistance information such as preferred resource, non-preferred or conflicted resources can be exchanged to prevent half duplex, hidden node and persistent collision issues. A common DRX cycle configuration allows Tx UE and Rx UE(s) to transmit and receive at the same time for all cast types. DRX cycle alignment in a relay architecture reduces latency and saves power by allowing Tx UE, Relay UE and Remote UE to transmit and receive data within the same DRX cycle. Defining different relaying architecture allows coverage extension for both gNB and sidelink devices. Reliability enhancement by avoiding packet loss can be achieved by forwarding packets to more than one Relay UEs.

\section{References}

[1] K. Ganesan, P.B. Mallick, J. Löhr, D. Karampatsis, A. Kunz, "5G V2X Architecture and Radio Aspects", 2019 IEEE Conference on Standards for Communications and Networking (CSCN), October 2019. 
[2] K. Ganesan, J. Löhr, P.B. Mallick, A. Kunz, R. Kuchibhotla, "NR Sidelink Design Overview for Advanced V2X Service", IEEE Internet of Things Magazine (Volume: 3, Issue: 1, March 2020).

[3] S. Husain, A. Kunz, A. Prasad, K. Samdanis, J. Song, "Overview of 3GPP Standards for Vehicle-to-Everything", IEEE Conference on Standards for Communications and Networking (CSCN), September 2017.

[4] W. Anwar, A. Traßl, N.Franchi, G. Fettweis, "On the Reliability of NR-V2X and IEEE 802.11bd", 2019 IEEE 30th Annual International Symposium on Personal, Indoor and Mobile Radio Communications (PIMRC), September 2019.

[5] Gaurang Naik, Biplav Choudhury, Jung-Min Park, "IEEE 802.11bd \& 5G NR V2X: Evolution of Radio Access Technologies for V2X Communications", IEEE Access, May 2019.

[6] 3GPP TS 22.186 "Enhancement of 3GPP support for V2X scenarios," v16.1, Dec. 2018.

[7] 3GPP TS 23.285 "Architecture enhancements for V2X services," v15.0.0, Mar. 2018.

\section{Biographies}

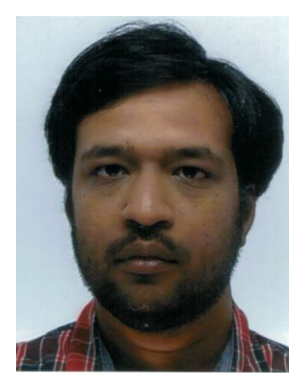

Karthikeyan Ganesan received MSC in Information Technology and communication engineering from the Tampere University of Technology, Finland in 2012. His previous work experience includes Layer1 firmware developer for GSM/EGPRS, LTE in various companies like Aricent, ST-Ericsson, CommAgility He started contributing to the $5 \mathrm{G}$ research at Huawei-Munich Research centre, Germany from 2015. Since 2019, he joined Lenovo Germany as member of the research and technology team, representing 
Lenovo/Motorola Mobility in the 3GPP RAN1 working group. His current focus area is NR-Sidelink design for vehicular communication and Industrial IOT.

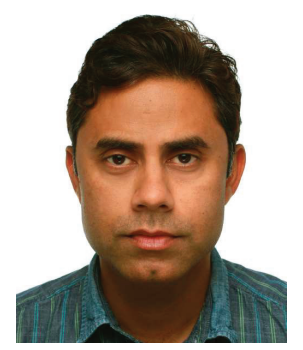

Prateek Basu Mallick received his MBA from Indian Institute of Management Bangalore, India in 2011. He started working with Hughes Software Systems, Gurgaon in 2000 after completing his engineer in Electronics and Telecommunication area. He later moved to Samsung India in 2004 where among other responsibilities he was involved in the commercialization aspects of many Samsung phone models and represented Samsung in 3GPP RAN2 until 2012. His focus areas are control plane and user plane aspects in features like Dual Connectivity, Carrier Aggregation, Vehicular and Sidelink communication etc. Since 2017, he is working in Lenovo Germany as a member of the research and technology team, representing Lenovo/Motorola Mobility in the 3GPP RAN2 and participating in other standards forum.

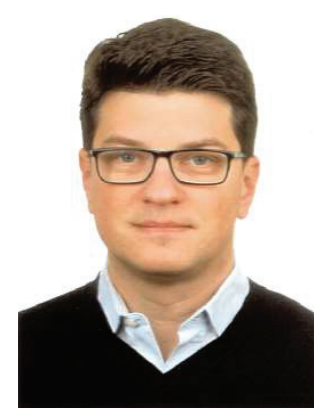

Joachim Löhr received his diploma degree in information technology and communications engineering from the Technical University of Darmstadt, Germany in 2002. Afterwards he joined the Panasonic R\&D Centre Germany 


\section{$90 K$ K. Ganesan et al.}

with focus on 3GPP standardization, mainly in the Radio Access Network working groups. From the beginning, he was a permanent member of the Panasonic 3GPP RAN1/2 delegation also later leading the Panasonic RAN2 delegation in the standard meetings. He was actively contributing to various topics from UMTS/HSPA (Rel-6/7) to the current 5G system (Rel-15). Since 2017, he joined Lenovo Germany as member of the research and technology team, representing Lenovo/Motorola Mobility in the 3GPP RAN2 working group. His current focus areas are operation in shared spectrum channel access, Industrial IOT and vehicular communication. 